\title{
Media de Ensambles y la No Extensividad en el Borde del Caos: Mapa de Kaplan-Yorke
}

\author{
Ensamble average and the nonextensivity in the edge of chaos: Kapla-Yorke Map
}

\author{
Garín F. Janampa Añaños ${ }^{1 *}$; Martin B. Sandoval Casas ${ }^{1,2}$; Juan M. Pesantes Rojas²; Reenaty A. Huatay \\ Enriquez $^{3}$. \\ *Autor de correspondencia
}

\begin{abstract}
Resumen
En el marco de la mecánica estadística no extensiva, son estudiadas numéricamente, por medio de medias de ensambles, la sensibilidad a las condiciones iniciales y la producción de la entropía por unidad de tiempo del mapa disipativo bidemsional de Kaplan-Yorke para dos casos: caos fuerte y caos débil (en el borde del caos). Se verifica que las propiedades de la sensibilidad y la producción de la entropía están relacionadas a un mismo valor del índice entrópico: q=1 para el caos fuerte y q $<1$ para el caos débil. Tambien se verifica, numéricamente, la generalización de la identidad de Pesin.
\end{abstract}

Palabras clave: Caos; sistemas dinámicos no lineales; mecánica estadística; mapas.

\begin{abstract}
In the statistic nonextensive mechanical framework, we numerically calculate, by ensemble average of the sensitivity to initial conditions and entropy production per unit time of bidimensional dissipative Kaplan-Yorke map, both for strong and weak (chaos threshold) chaotic cases. We verify that sensitivity and entropy production properties are related to one and same value of entropic parameter: $\mathrm{q}=1$ for strong chaos and $\mathrm{q}<1$ for weak chaos. We also numerically verify the generalization of Pesin identity.
\end{abstract}

Keywords: Chaos; nonlinear dynamical systems; statistical mechanics; maps.

\section{Introducción}

Una gran variedad de fenómenos naturales muestran un comportamiento complejo, impredecible y aparentemente aleatorio. Los ejemplos conocidos son el flujo turbulento de una corriente de un fluido, la variación del clima, el comportamiento anómalo de los precios de las acciones en la bolsa de valores, por mencionar algunos. El paradigma para esta clase de fenómenos macroscópicos es el problema del flujo turbulento en los fluidos (Eckmann, 1981). Otros ejemplos de comportamiento irregular y complejo, son aquellos que ocurren en la dinámica de las moléculas y los átomos en un gas de partículas cargadas, o sea el plasma. Estos sistemas microscópicos (Mazur, 2017) definen otra clase de problemas físicos importantes que presentan una interrogante aun no resuelta (Baranger, 2000): ¿Cómo es que los movimientos determinísticos y reversibles de partículas individuales pueden causar un comportamiento impredecible e irreversible del sistema?, como es descrito por la mecánica estadística y la termodinámica.

Si bien es cierto que la física ha realizado grandes avances en los últimos cien años, las descripciones teóricas de estos fenómenos complejos presentan problemas aún por resolver. La dificultad principal se encuentra en el carácter no lineal de las ecuaciones de Navier-Stokes para los fluidos y las ecuaciones de Newton para tres o más partículas que interactúan (Arnold, 1978). Debido a esta no linealidad, es muy difícil hacer predicciones para el comportamiento de los sistemas estudiados. La masificación del uso de los ordenadores marca una línea divisoria en el estudio de los sistemas dinámicos. Con el computador se puede simular y resolver ecuaciones, calculando sus soluciones a partir de las condiciones iniciales y valores de los parámetros deseados. La recolección de los resultados numéricos obtenidos ayuda a desarrollar una intuición sobre los posibles comportamientos que esas ecuaciones presentan. Como consecuencia, en los últimos treinta años se obtuvo grandes progresos, usando una síntesis única de simulaciones numéricas y de aproximaciones analíticas. Esta nueva estrategia, que combina simulaciones numéricas y el análisis matemático, dio origen a una nueva rama interdisciplinaria dentro de la dinámica de sistemas no lineales (Baranger, 2000; Dorfman, 1999). El trabajo realizado en esta rama no solamente fue aplicado a la física, sino que también a una gran variedad de problemas no lineales en otras áreas científicas, como la evolución de las reacciones químicas, del control en la generación de circuitos eléctricos, de la interacción de las poblaciones biológicas, de 
la respuesta de las células cardiacas a los impulsos eléctricos y las fluctuaciones del precio de las acciones en las bolsas de valores. Los especialistas en dinámica no lineal usan la palabra "caos" como un término técnico con un significado matemático preciso para definir un comportamiento irregular e impredecible del sistema que es determinístico y no lineal (Baranger, 2000). Isaac Newton creía que las ecuaciones de la mecánica clásica abarcaban solamente a un universo ordenado y regular, sin embargo, hoy en día se sabe que tal afirmación no es verdadera.

Los sistemas disipativos sencillos pueden presentar una estructura matemática rica que se traduce en una diversidad de comportamientos físicos que son muchas veces observados en los experimentos (May, 1976; Mazur, 2017). Los mapas no lineales de bajas dimensiones juegan un rol importante en el desarrollo de la teoría del caos, tanto en la física, matemática y en la ingeniería (Beck y Schologl, 1993). Estos mapas muestran diferentes clases de universalidad (Hilborn, 1994; Cvitanovic, 1984), Marcantoni et al. (2017). En particular los mapas en bajas dimensiones, como la de Kaplan-Yorke, son modelos paradigmáticos que sirven para estudiar la emergencia de comportamientos complejos en sistemas dinámicos (Kaplan y Yorke, 1979). En esta área fueron desarrollados muchos trabajos con el objetivo de encontrar propiedades del caos que pudiesen dar una clasificación de los sistemas determinísticos (véase Cvitanovic, 1984). En particular, fueron desarrollados indicadores dinámicos tales como la sensibilidad a las condiciones iniciales, los exponentes de Lyapunov, la entropía de Kolmogorov-Sinai (May, 1976; Pesin, 1977; Kolmogorov, 1958), la entropía topológica (Bowen, 1973) entre otros. Sin embargo, sabemos hoy en día de la existencia de sistemas dinámicos naturales y artificiales para los cuales todos los indicadores conocidos no detectan caos, pero los resultados numéricos muestran un comportamiento complejo de la evolución temporal de estos sistemas (Korabel y Barkai, 2010; Chowdhury and Swingle, 2017). Este es el caso típico de las manifestaciones no conocidas de los sistemas en la frontera entre el caos y las orbitas regulares. Este comportamiento complejo y determinístico fue denominado caos débil, Zaslavsky y Edelman (2001); Zaslabsky et al. (1991), para poder distinguirlo del caos propiamente dicho, que hoy en día se le conoce como caos fuerte; es decir del comportamiento de los sistemas dinámicos con entropía métrica positiva o exponentes de Lyapunov positivos. En el caos débil los exponentes de Lyapunov y la entropía métrica son nulos. Este fenómeno condujo a tentativas de generalización de estos indicadores dinámicos (Gell-Mann y Tsallis, 2004; Korabel y Barkai, 2009, (2010).

\section{Materiales y Métodos}

\section{Materiales: Mapa de Kaplan-Yorke}

El mapa de Kaplan-Yorke pertenece a la clase de mapas disipativos bidimensionales. Una de las características peculiares de este sistema es el hecho de que su significado físico es directo. Este punto distingue a este mapa bidimensional de otros mapas conocidos tales como el mapa de Henon entre otros (Beck,1993; Hilborn, 1994).

Si consideramos una partícula de masa 1 que se mueve bajo la influencia de una fuerza puntual.

$$
\vec{L}_{\tau}(t)=\sum_{n=0}^{\infty} \eta_{n} \delta(t-n \tau)
$$

donde $t$ es la diferencia de tiempo entre los impulsos (se supone que los impulsos son equidistantes). $\eta_{n}$ es la intensidad del impulso en el tiempo $\eta \tau$. Si el movimiento de la partícula sucede en un medio viscoso, adicionalmente emerge una fuerza de fricción ejercida sobre la partícula. $\mathrm{Si}$ esta fuerza es proporcional a la velocidad de la partícula $\vec{y}$ (t), la ecuación de movimiento es

$$
\frac{d \vec{Y}(t)}{d t}=-\gamma \vec{Y}(t)+\vec{L}_{\tau}(t)
$$

donde $\gamma$ es la viscosidad del líquido. A este nivel se supone la existencia de un sistema dinámico $\mathrm{T}$ con tiempo discreto (Beck, 1993): $X \rightarrow X \subset R^{k}$ es una función $f: X \rightarrow R^{m}(\vec{y}(\mathrm{t})$ toma valores en $R^{m}$ ), tal que

$$
\eta_{n}=f\left(x_{n-1}\right)
$$

La solución $\vec{y}(\mathrm{t})$, de la ecuación (2.2) puede ser representada

$$
\vec{Y}(t)=e^{-\gamma(t-n \tau)} y_{n}, \quad\left(n=\left[\begin{array}{l}
t \\
\tau
\end{array}\right]\right)
$$

$x_{n+1}=T x_{n}$

dondi $\left[\frac{t}{\tau}\right]$ considera solamente la parte entera, por otro lado, $y_{n}$ es obtenida por la relación de iteración $\mathrm{U}$ :

$$
\begin{aligned}
& x_{n+1}=T x_{n} . \\
& y_{n+1}=\lambda y_{n}+f(x)
\end{aligned}
$$

donde $\lambda=e^{-\gamma \tau}$ es un parámetro.

Con esta cosideración el sistema dinámico (6) con tiempo discreto tiene una interpretación física directa (Beck,1993): $y_{n}=Y(n \tau)$ es la velocidad de la partícula con amortiguación puntual. Considérese que la velocidad inicial $y_{0}$ es fija. Si ademas fijamos la posición inicial $X_{0}$, la evolución temporal de la velocidad es determinada para todos los tiempos. Alternativamente $X_{0}$, puede ser considerado como una variable aleatoria con una cierta distribución de probabilidades $\mu\left(X_{0}\right)$ De esta manera, $\vec{L}_{\tau}(\tau)$ es por definición un proceso estocástico y la ecuación (2) es una ecuación diferencial estocastica (Van Kampen, 1981; Schuss, 1980; Friedmann, 1975). De acuerdo con el teorema de la existencia de Kolmogorov (Friedmann, 1975) para todo proceso estocástico existe un espacio de fases X, un sistema dinámico $\mathrm{T}$, un mapa $\mathrm{f} \mathrm{y}$ una medida de probabilidades $\mu$ tales que $\eta_{n}=f\left(\mathrm{~T}^{\mathrm{n}-1} \mathrm{x}_{0}\right)$ En este sentido la ecuación (2) y el sistema dinámico (6) son equivalentes. De esta manera 
obtenemos el mapa de kaplan-Yorke (Kaplan y Yorke, 1979) el mismo que fué escrito para $T x=1-2 x^{2}, f(x)=x$. Para este trabajo se elige la siguiente estructura del mapa (6)

$x_{n+1}=1-a x_{n}^{2}$

$Y_{n+1}=\lambda y_{n}+x_{n}$

Para el mapa (7) se cumple los siguientes teoremas demostrados en Beck, 1990: (I) Si el comportamiento a lo largo del tiempo (lo suficientemente grande) de la fuerza puntual es periódica, entonces la velocidad de la partícula también es periódica; (II) si la fuerza puntual que actúa sobre la partícula es ergódica la velocidad de esta también es ergódica; (III) si la fuerza puntual que actúa sobre la partícula exhibe un comportamiento "mixto" entonces la velocidad de esta tiene un comportamiento "mixto" a lo largo del tiempo. Como consecuencia del teorema I el mapa (7) de dos dimensiones muestra bifurcaciones sub armónicas para los mismos valores del parámetro de dirección $\alpha$ del sistema $\mathrm{T}$ es decir de $x_{n+1}=1-a x_{n}{ }^{2}$ independiente de $\lambda$. Así para ambas ecuaciones (7) el punto de acumulación de las bifurcaciones es la constante de Feigenbaum $\delta$. De aquí, se puede deducir que los parámetros $\alpha$ y $\lambda$ están desacoplados. De esta manera podemos afirmar que el valor crítico del parámetro de dirección, que indica el borde del caos, es el mismo que del mapa logístico (Beck, 1993), exactamente exactamente $a=a_{\mathrm{c}}=1.401155189 .$.

\section{Metodología}

\section{Sensibilidad a las condiciones Iniciales}

Primeramente analicemos el concepto de la sensibilidad a las condiciones iniciales para sistemas de una dimensión (Latora, Rapisarda y Ruffo, 1998; Beck, 1993; Beck, 2001). La función de la sensibilidad a las condiciones iniciales se define como

$$
\xi(t) \equiv \lim _{\Delta x \rightarrow 0} \frac{\Delta x(t)}{\Delta x(0)}
$$

donde $\Delta \mathrm{x}(0)$ es la discrepancia de las condiciones iniciales en el tiempo $t=0$, y $\Delta x(t)$ es su dependencia temporal. La función exponencial

$$
\xi(t)=\exp \left(\lambda_{1} t\right)
$$

con, conocido como el exponente de Lyapunov, satisface la ecuación diferencial

$$
\frac{d \xi}{d t}=\lambda_{1} \xi
$$

Si $\lambda_{1}<0\left(\lambda_{1}>0\right)$, se dice que el sistema es fuertemente insensible (sensible) a las condiciones iniciales. Por otro lado, si $\lambda_{1}>0$ (como sucede en el borde del caos), entonces se espera que la función $\xi(t)$ satisfaga a la ecuación diferencial

$$
\frac{d \xi}{d t}=\lambda_{q} \xi^{q}
$$

La misma que contiene el denominado exponente-q generalizado de Lyapunov $\lambda_{q}$, propuesto por vez primera en Tsallis et al. (1997). La función exponencial-q, solución de la ecuación (11) recupera el caso estándar (9) para q $=1$, mientras que cuando $q \neq 1$ la exponencial-q muestra un comportamiento del tipo de ley de potencias. Si Si $q<1$ $(q>1)$ y $\lambda_{q}>0\left(\lambda_{q}<0\right)$, el sistema es débilmente sensible (insensible) a las condiciones iniciales. Por otro lado la función logarítmica-q inversa a la exponencial-q se escribe de la siguiente manera:

$\ln _{q} \equiv \frac{x^{1-q}-1}{1-q}$

Naturalmente que cuando $\mathrm{q}=1$ se tiene tiene $\ln =\ln x(\mathrm{x})$. Si $\mathrm{x} \rightarrow 1$ tenemos que $\ln _{\mathrm{q}} x \sim 1-x(\forall x)$.

Para la sensibilidad, procedemos como sigue: consideremos en una posición inicial de $x$ y $y$, para el mapa (7), dos puntos muy próximos para ambas variables, con una distancia inicial de $\Delta x(0)=10^{-12}$ y $\Delta y(0)=10^{-12}$ para $x$ y $y$ respectivamente; luego se calcula numéricamente $\xi(t)$ a partir de su definición. Repetimos muchas veces esta acción partiendo de valores de $x$ y de $y$ escogidos aleatoriamente en el intervalo $[-1,1]$. Finalmente calculamos la media del logaritmo-q de la función de la sensibilidad $\ln _{q} \xi$, extrayendo así la media global (del orden de $10^{7}$ condiciones iniciales) del comportamiento, haciendo realizaciones aleatoriamente en todo el espacio de fases [-1,1].

\section{La Generalización de la Entropía de Kolmogorov-Sinai y la Identidad de Pesin}

Para sistemas dinámicos que presentan caos, la entropía de Kolmogorov-Sinai (KS) (Kolmogorov, 1958; Sinai, 1959) puede ser definida como el incremento por unidad de tiempo de la entropía estándar de Boltzmann-Gibbs (BG):

$$
S_{1}=-k_{B} \sum_{\mathrm{i}=1}^{\mathrm{w}} p_{i} \ln p_{i} \quad\left(k_{B} \equiv 1\right)
$$

Se sabe que la entropía KS está íntimamente ligada a la identidad de Pesin (Pesin, 1977) que postula: $K_{1}=\lambda_{1}$ si $\lambda_{1}>0$ $\mathrm{y} \mathrm{K}_{1}=0$ para otras variantes. Por otro lado, es conocido que la entropía KS puede ser definida en términos de una única trayectoria en el espacio de fases, usando la representación simbólica de las regiones del espacio de fases particionado (Hedlund y Morse, 1990). Esta definición puede ser sustituida de manera equivalente por una basada en un ensamble de condiciones iniciales (Ananos y Tsallis, 2004). Esta versión es la que emplearemos en este trabajo. Los casos marginales, es decir, aquellos en las cuales $\lambda_{1}=0$, incluyen las bifurcaciones con periodo doble y tangencial, así como el borde del caos. Para estos casos, se introdujo una versión generalizada en Tsallis (1988), Tsallis et al. (1997), de la entropía KS como la tasa de incremento de la entropía no extensiva:

$$
S_{q}(t)=k_{B} \frac{1-\sum_{i=1}^{W}\left[p_{i(t)}\right]^{q}}{q-1} \quad\left(k_{B} \equiv 1\right)
$$

Esta entropía no extensiva permite una generalización de 
la mecánica estadística de Boltzmann-Gibbs la misma que se recupera cuando cuando $q \rightarrow 1\left(S_{1}=-k_{B} \sum_{i=1}^{W} p_{i} \ln p_{i}\right)$. Una revisión de las propiedades de esta entropía y tópicos relacionados se puede encontrar en Salinas y Tsallis (1999), Abe y Okamoto (2004) y Gell-Mann y Tsallis (2004). De esta manera se postula la siguiente versión generalizada de la entropía KS (Tsallis et al. (1997).

$$
K_{q}=\lim _{t \rightarrow \infty} \lim _{W \rightarrow \infty} \lim _{N \rightarrow \infty} \frac{\left\langle s_{q}\right\rangle(t)}{t}
$$

donde $\mathrm{t}$ es el tiempo, $\mathrm{W}$ es el número de regiones en la partición del espacio de fases y $\mathrm{N}$ es el número de condiciones iniciales que evolucionan con el tiempo. Se espera que se cumpla la identidad de Pesin, es decir $K_{q}=\lambda_{q}$ si $\lambda_{q}>0$ caso contrario $K_{q}=0$. El análisis de la tasa de crecimiento de la entropía ofrece otra manera de caracterizar el índice entrópico especial $q_{\text {sen }}$ en el borde del caos. El procedimiento numérico, desarrollado en este trabajo, fue introducido por primera vez en Latora y Baranger (1999) para sistemas conservativos y luego para sistemas disipativos en una dimensión en Latora et al., 2000.

Para el mapa (7) procedemos a dividir el intervalo [-1,1] en $\mathrm{W}$ intervalos. Luego elegimos aleatoriamente $\mathrm{N}$ condiciones iniciales con un valor fijo del parámetro de control $\alpha$. En cuanto t comienza a evolucionar estos $\mathrm{N}$ puntos comienzan a diseminarse dentro del espacio de fase $[-1,1]$, obteniéndose así un conjunto $\left\{N_{i}(t)\right\}$ con $\sum^{\mathrm{w}}{ }_{\mathrm{i}=1} N_{i}$ $(t)=N, \forall t$, que consecuentemente involucra un conjunto de probabilidades $\left\{p_{i}(\mathrm{t}) \equiv N_{i}(t) / N\right\}$. Luego se escoge un valor para q y se calcula $S_{q}(t)$ haciendo uso de la ecuación (4.2). Finalmente después de realizar una media de muchas condiciones iniciales escogidas aleatoriamente $\left\langle S_{q}\right\rangle(t)$ en $(t)$ en todo el espacio de fases se calcula numéricamente (15).

Nótese que el procedimiento de las medias, por un lado, es un procedimiento termo-estadístico y, por otro lado, es una manera natural de minimizar las fluctuaciones que aparecen en el borde del caos (Ananos y Tsallis, 2004). Y son estas fluctuaciones las que dificultan la caracterización del índice entrópico para la cual $\left\langle S_{q}\right\rangle(t)$ tiene un crecimiento lineal.

\section{Resultados y discusion}

Se ha confirmado numéricamente, para el mapa (7), que el comportamiento exponencial, se obtiene para valores específicos del parámetro de control $a \neq a$, en particular para $a=2.0$. Mientras que para el borde del caos $a=a_{c}$, la perdida de la ergodicidad se reemplaza con la aparición del comportamiento del tipo de ley de potencias (GaggeroSager et al., 2011). Estas dos afirmaciones son corroboradas numéricamente a través del método de la función de la sensibilidad a las condiciones iniciales y el método de la entropía métrica KS. Los resultados numéricos son mostrados en la Figura1 A,B y Figura 2A, B para el caos fuerte y para el borde del caos (caos débil) respectivamente. El procedimiento desarrollado es el mismo que en Ananos y Tsallis (2004). Cuando los experimentos numéricos, para la sensibilidad a las condiciones iniciales, son ejecutados para valores críticos del parámetro de control $\left(a_{c}=\right.$ $1.401155189 \ldots$... el incremento lineal de $\langle\ln q \xi\rangle$ (t) sucede sólo para determinados valores específicos del índice entrópico q el cual lo denotamos como $q^{a v}{ }_{\text {sen }}$ donde av indica el procedimiento de las medias y sen se refiere a la función de la sensibilidad. En el borde del caos $a=a_{c}=$ 1.401155189 el índice entrópico relevante es $q=0.36 \pm 0.01$, (ver Figura2A, en contraste para el caos fuerte $q=1$ (Figura1A). Con este valor la curva de la sensibilidad a las condiciones iniciales tiene un crecimiento lineal antes de la saturación debido al número finito de las condiciones iniciales. La inclinación tiene un valor de 0.29 cuyo valor coincide con el valor de la inclinación de la curva de la producción de la entropía (Figura 2B), corroborándose la identidad de Pesin.

Los resultados numéricos para la entropía $\mathrm{KS}$, con el procedimiento de la media de ensambles, confirman la identidad de Pesin: exactamente $K_{q_{s e n}^{a v}}=q_{\lambda_{s e n}^{a v}}$ Este resultado se muestra en las Figura 1B y Figura 2B para el caos fuerte y para el borde del caos $\left(a=a_{c}=1.401155189\right)$ respectivamente. Los resultados numéricos para estos dos regímenes, caos fuerte y débil (borde del caos), del mapa (7) muestran un crecimiento lineal en el tiempo antes de llegar a un estadio de saturación (de forma análoga para la sensibilidad véase la Figura 1A y Figura 2A) debido al valor finito de las particiones $\mathrm{W}$ que se efectúa en el espacio de fases. La inclinación de la parte lineal de la entropía, la producción de la entropía, es numéricamente igual a $K_{1}=0,69 \pm 0.01$ para el caos fuerte $(\alpha=2.0)$ que coincide con el valor obtenido con el método de la sensibilidad a las condiciones iniciales (exponentes de Lyapunov $\lambda_{1}=0.69$ ). De esta manera se corrobora la identidad de Pesin para el caos fuerte. En la Figura (2B) se muestra los resultados para el borde del caos, es decir cuando $a=a_{c}=1.40115589 \ldots$ La curva de la entropía se obtiene después de una media típica de $5 \times 10^{5}$ realizaciones implementadas aleatoriamente. Esta curva es lineal en el intervalo de tiempo correspondiente para $q=q{ }^{a v}{ }_{s e n}=0.36 \pm 0.01 \mathrm{y}$ desaparece para $\forall q>0.36$ y diverge para $\forall q<0.36$. La identidad de Pesin también es corroborada para el borde del caos: $K_{0.36}=0,29 \pm 0.01$ que coincide con la inclinación de la curva de la función de la sensibilidad a las condiciones iniciales $\lambda_{0.36}=0,29 \pm 0.01$.

Con el fin de hacer más convincentes los resultados arriba mencionados, se ajusta las curvas de producción de la entropía $\left\langle S_{q}\right\rangle(t)$ con el polinomio $S(t)=a+b t+c t^{2}$ en el intervalo $\left[t_{1}, t_{2}\right]$ (Latora y Baranger, 1999; Ananos y Tsallis, 2004). Entonces se define $R=c\left(t_{1}, t_{2}\right) / b$, como una medida de la importancia del término no linea 1 en el ajuste realizado en todas las curvas analizadas, tanto para la entropía como para función a la sensibilidad a las condiciones iniciales. Si los puntos de una curva estuviesen sobre una línea recta perfecta, $\mathrm{R}$ debería ser cero. Para el mapa de Kaplan-Yorke, en el borde del caos, por ejemplo, se escoge $\mathrm{W}=800 \mathrm{X} 800$ (Tsallis et al., 1997; Latora $\mathrm{y}$ Baranger, 1999). Si el valor de W aumenta el intervalo temporal a considerarse aumenta. 

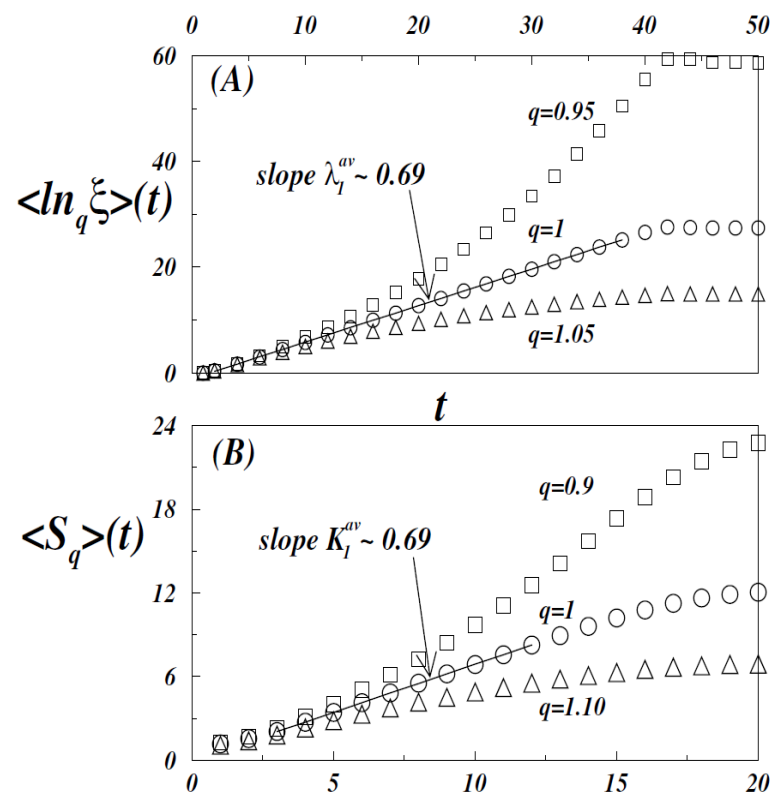

Figura 1 (A,B). Dependencia temporal de $\left\langle\ln q \xi\right.$ y $\left\langle S_{q}>(t)\right.$ para el mapa de Kaplan-Yorke con el parámetro de control $\alpha=2.0, \mathrm{y} \quad 0<\lambda \leq 1$. Se considera tres valores diferentes de $q$ . Las curvas son el resultado de la media sobre (A) $10^{7}$ realizaciones con una discrepancia de $\Delta x(0)=10^{-12}$ de las condiciones iniciales en $t=0$ en (B) 6000 realizaciones con $\mathrm{W}=800 x 800$ y $\mathrm{N}=10 \mathrm{~W}$.

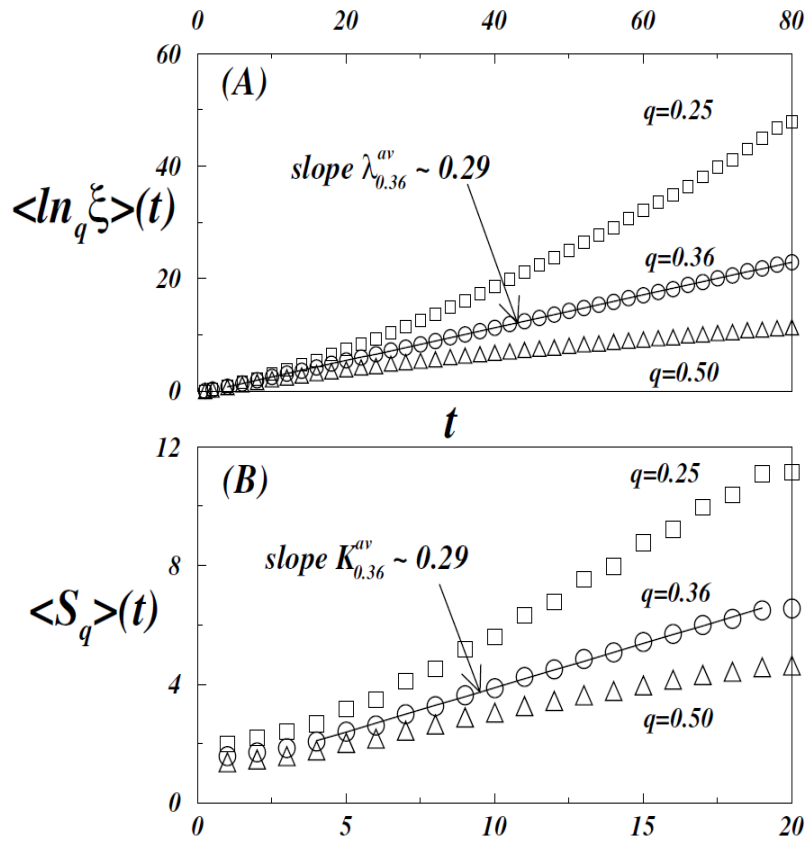

Figura 2 (A,B). Dependencia temporal de $\left\langle\ln q \xi\right.$ y $\left\langle S_{q}>\right.$ $(t)$ para el mapa de Kaplan-Yorke con el parámetro de control $\alpha_{\mathrm{c}}=1.401155189$, y $0<\lambda \leq 1$. Se considera tres valores diferentes de $q$. Las curvas son el resultado de la media sobre (A) $10^{7}$ realizaciones con una discrepancia de $\Delta x(0)=10^{-12}$ de las condiciones iniciales en $t=0$ en (B) 60000 realizaciones con $W=800 \times 800$ y $N=10 \mathrm{~W}$

\section{Conclusiones}

Dentro del marco de la estadística no extensiva los resultados del presente trabajo son las siguientes: (I) Para el mapa disipativo bidimensional de Kaplan-Yorke (7) se realizó la media de ensambles para la sensibilidad y la producción de la entropia con la finalidad de determinar el índice entrópico $q^{a v}{ }_{\text {sen }}$, siendo este el mismo con los dos métodos; (II) Para el caos fuerte se verifica que $q^{a v}{ }_{s e n}=1$ y $K^{a v}{ }_{\text {sen }}=\lambda_{\text {sen }}=0.69$, En el borde del caos $q^{a v}{ }_{\text {sen }}=0.36 \mathrm{y}$ $K^{a v}{ }_{\text {sen }}=\lambda_{\text {sen }}=0.29$, osea diferente de cero como usualmente se puede encontrar en la literatura de sistemas dinámicos no lineales (Hilborn, 1994; Beck, 1993; Ott,1993). De esta manera se verifica, numéricamente, la generalización de la identidad de Pesin para el caso del mapa disipativo bidimensional de Kaplan-Yorke, para ambos casos: caos fuerte y para el borde del caos (caos débil).

\section{Literatura citada}

Abe, S. and Okamoto, Y. 2001. Nonextensive Statistical Mechanics and its Applications, Series Lectures Notes in Physics: Springer-Verlag, Berlin.

Ananos, J. G. and Tsallis, C. 2004. Phys. Rev. Lett. 93, 020601 .

Arnold, V. I. 1978. Mathematical Methods in Classical Mechanics. Springer, New York.

Baranger, M. 2000. Complexity Chaos, Entropy. New England Complex Systems Institute.

Beck, C. and Swinney, H. L. 2001. Phy. Rev. E 63, 035303.

Beck, C. and Schlogl, F. 1993. Thermodynamic of Chaotic Systems Cambridge University Press, Cambridge.

Beck, C. 2001. Phys. Rev. Lett. 87, 180601.

Beck, C., Ergodic. 1990. Properties of a Kicked Damped Particle. Commum. Math. Phys. 130, 51-60.

Bowen, R. 1973. Topological entropy for noncompact sets, Trans. AMS 84,125- 136.

Chowdhury, D. and Swingle, B. 2017. Phys. Rev. D 96, 065005 .

Cvitanovic, P. 1984. Universality in Chaos A. Hilger, Bristol, (1984).

Dorfman, J.R. 1999. An introduction to chaos in Nonequilibrium Statistical Mechanics. Cambridge University Press. Cambridge.

Eckmann, J.P. 1981. Rev. Mod. Phys. 53 (4): Part I.

Friedman, A. 1975. Stochastic differential equations and applications. New York, Academic Press.

Gaggero-Sager, L.M.; Pujal, E.R. and Sotolongo-Costa, O. 2011. Phys. 41: 297-303.

Gell-Mann, M. and Tsallis, C. 2004. Nonextensive EntropyInterdisciplinary Applications, Oxford University Press, New York.

Hedlund, G.A. and Morse, M. 1990. Symbolic dynamics, Am. J. Math. 60, 815-866.

Hilborn, R. C. 1994. Chaos and Nonlinear dynamics, 
Oxford University Press, New York.

Kaplan, J.L. and Yorke, J.A. 1979. Lecture notes in mathematics 730, p. 204. Berlin, Heidelberg, New York, Springer.

Kolmogorov, A.N. 1958. Dok. Acad. Nauk. SSSR 119, 861.

Korabel, N. and Barkai ,E., 2009. Phys. Rev. Lett. 102, 050601.

Korabel, N. and Barkai, E. 2010. Phys. 2010. Rev. E 82 19, 016209.

Latora, V.; Rapisarda, A; and Tsallis, C. 2001. Phys. Rev. E 64, 056134.

Latora, V.; Rapisarda, A. and Ruffo, S. 1998. Phy. Rev. Lett. 80692.

Latora, V. and Baranger, M. 1999. Phy. Rev. Lett. 82520.

Latora, V.; Baranger, M., Rapisarda, A., Tsallis, C. 2000. Phys. Lett. A 273, 97.

Marcantoni, S.; Alipour.; Benatti, F.; Floreanini, R.; Rezakhani, A. T. 2017. Sci. Rep. 7, 12447.

May R. 1976. Simple mathematical Models with very complicated dynamics, Nature, 261, 149.

Mazur, A. 2017. Phys. Rev E95, 062417.

Ott, E. 1993. Chaos in dynamical system, Cambridge University Press.

Pesin Ya, Russ. Math. 1977. Surveys 32, 55.

Salinas, S.R.A and Tsallis, C. 1999 Braz. J. Phys. 29, 1.

Schuss, Z. 1980. Theory and applications of stochastic differential equations. New York, Wiley.

Sinai, Y. G.1959 Dok. Acad. Nauk. SSSR 124, 768.

Tsallis, C.; Plastino, A. R. and Zheng, Z-W. 1997. Chaos, Solitons and fractals 8,885 .

Tsallis, C. J. 1988. Stat. Phys. 52, 479-87.

Van Kampen, N.G. 1981. Stochastic processes in physics and chemistry. Amsterdam, North-Holland.

Zaslavsky, G.M.; Edelman, M. 2001. Weak mixing and anomalous kinetics along filamented surfaces. Chaos, 11(2).

Zaslavsky, G.M.; Sagdeev, R.Z.; Usikov, D.A. and Chernikov, A.A. 1991. Weak chaos and quasi-regular patterns, Cambridge University Press. 